\title{
Treatment of Transverse Olecranon Fractures: Comparison Between Tension Band Wiring And Transcortical Screw
}

\author{
Dr. T.D. Bhattacharyya ${ }^{1}$, Dr. H.C. Frank ${ }^{2}$, Dr. Kishore Hazarika ${ }^{3}$, \\ Dr. Saksham Mittal ${ }^{4}$ \\ (Department of Orthopedics, Gauhati Medical College)
}

\begin{abstract}
:
Aim: The aim of this study is to compare the clinical and functional outcomes of transverse olecranon fractures treated with tension band wiring and transcortical screw.

Materials and Methods: A prospective randomized controlled trial study was carried out for a total of 40 cases of transverse olecranon fractures aged between 18-70 years. After initial work up the patients underwent surgery by either of two techniques. All patients were followed up for a minimum period of 6 months and the results were assessed radiologically and clinically using Mayo Elbow Performance Score.

Result: The average range of motion in patients treated with $T B W$ was found to be $105.5^{\circ}$ as compared to screw fixation, which was 100.1 $1^{\circ}$ MEPS showed satisfactory results (good + excellent) in $90 \%$ cases treated with $T B W$ in comparison to $75 \%$ cases treated with screw fixation. The average time taken for union in tension band wiring group was 10.15 weeks and that for screw fixation was 10.6 weeks.

Conclusion: Tension band wiring and parallel screw fixation are equally effective method of fixation in terms of union and elbow movements. However post operative patient satisfaction was found to be more in screw fixation technique though the procedure requires more accuracy.
\end{abstract}

Keywords: Tension band wiring, Transcortical screw, MEPS

\section{Introduction}

The fracture of the olecranon is an intra-articular injury with loss in the extension mechanism of the elbow joint; therefore its treatment is always operative ${ }^{1}$. Fractures about the elbow represent approximately $5.5 \%$ of fractures of the entire skeleton of which radial head fractures are seen most frequently (2.8\%), followed by fractures of the olecranon and radial neck ( $1 \%$ each), the distal humerus $(0.5 \%)$, and extra-articular fractures of the proximal radius and ulna $(0.2 \%)^{2}$. The common causes of Olecranon fractures are mostly due to self-fall, motor vehicle crash, fall from a height, or a direct blow to the elbow and are commonly observed orthopedic injuries in the emergency room $^{3}$ Different classification methods are present to assess the fracture patterns, among which Mayo's classification system is considered to be the most commonly used. They depend on the degree of fracture displacement, the stability of the elbow joint and communition at the fracture site. Undisplaced fractures are Mayo Type I fractures and these are exceptional and can be managed conservatively. However, most olecranon fractures are displaced, considered as Mayo Type II and Type III, and in such cases operative treatment is recommended ${ }^{4}$.

Unstable fractures of olecranon, which are angulated, rotated or shortened, require surgical fixation for early recovery and functional improvement. The main objective of operation is to achieve adequate reduction and rigid fixation, allowing early mobilization in an attempt to reduce morbidity due to stiffness ${ }^{5}$. The primary objective of operative treatment of olecranon fractures is anatomic restoration of the trochlear notch.

Weber and Vasey in 1963 introduced tension band wiring for its treatment but several modification attempts were undertaken, especially to avoid migration of the Kirschner wires ${ }^{5}$. Fractures can be approached by an open reduction and fixation using lag screws, plates, and interosseous wires ${ }^{6 .}$

The AO tension band technique provides strong fracture fixation in terms of low non-union rate but has other common complications. The most common of these complications is hardware prominences caused by backing out and protrusion of the proximal ends of the $\mathrm{K}$-wires through the thin soft tissues covering the extensor surface of the elbow. However Tyler $e t a .^{7}$ advocated the use of an alternate fixation method which have equal stability and less hardware complications as compared to the $\mathrm{AO}$ technique. He used threaded cancellous screws for this purpose. It seems likely that the threads of cancellous screws would make them less likely to back out as compared with smooth K-wires.

The purpose of the present work is to critically examine and document two widely applied technologies transcortical screw fixation and tension band wiring in transverse olecranon fracture in terms of anatomic restoration, functional outcome and complications. 
Treatment of Transverse Olecranon Fractures: Comparison Between Tension Band Wiring And..

\section{Aim And Objective:}

The aim of this study is to compare the clinical and functional outcomes of transverse olecranon fractures treated with Tension band wiring versus Transcortical Screw fixation and to highlight their advantages and disadvantages.

\section{Materials And Method:}

A prospective randomised controlled trial study was carried out for a total of 40 cases of transverse olecranon fractures aged between 18yrs - 70yrs attending the OPD and Emergency department of Orthopaedics, Gauhati Medical College \& Hospital .The study was conducted for a period of 16months from $1^{\text {st }}$ May 2014 to $15^{\text {th }}$ september 2015 .

All the patients in this study were admitted either through out-patient department or through emergency department of the college. On receiving the patient, initial symptomatic treatment and a detailed history was taken. A thorough clinical examination was carried out to exclude any associated injury \& other medical and surgical illness which may require necessary treatment. Injured limb was examined thoroughly for open fractures, distal neurovascular status and to exclude compartment syndrome. The limb was initially immobilized with a long arm/above elbow posterior POP slab. Initial radiographs were done taking true antero-posterior and lateral views of the elbow. The fracture pattern was then classified according to the AO classification. At this stage, randomization was done using the plan generated from the website www.randomization.com to allocate the patient into one of the following two treatment model -

1. Tension Band wiring

2. Transcortical screw fixation

In all the patients who gave consent for operation, routine investigations were done and operation performed. The limb was kept elevated in posterior slab for the first two days. After subsidence of swelling and subjective decrease in pain the slab was removed and put on an arm pouch. With this, gradual flexion extension exercises were started within the limit of tolerance of pain. The patients were then discharged and called for follow up and suture removal at $10-14^{\text {th }}$ post operative day. The subsequent follow up was at 6,12 and 18 weeks after discharge and then subsequently every 2 months. All patients were followed up for atleast 6 months.

Radiological, clinical and functional assessment was done using Mayo Elbow Performance Score.

Statistical analysis was done using suitable biostatistical technique on each variable in the same patient and between two treatment groups. The software Instat Graphpad3 and R3.0.1 were used in the analysis. P value less than $0.5 \%$ was considered significant.

\section{Observation And Results}

The average age of the patients treated in TBW group and screw fixation group was 43.3 vs 42.9 which was statistically insignificant. In TBW group, there were 14 males and 6 females \& in screw fixation group there were 12 males and 8 females. The fractures were more commonly encountered on the Right (70\%). There were no bilateral cases. The commonest mode of injury in our patient was fall on ground (57.5\%).

Most of the cases $(70.0 \%)$ were operated in 3-7 days following injury. The mean time interval between surgery and trauma was 6.2 days. The operative time was calculated from starting of the skin incision till last skin stitch was made. The mean operative time of surgery in the TBW group was found to be 30 minutes, (standard deviation is1.97). The mean operative time of surgery in the Screw fixation group was found to be 45 minutes, (standard deviation 2.271). The average operative time of surgery in both the groups was 37.5 minutes with a standard deviation of 7.88. On statistical analysis of the mean operating time of the two groups, the $p$ value was found to be $<0.05$ (unpaired t test:), which is statistically insignificant.

Mean range of motion in our study was 102.8 degrees. In TBW group it was 105.5 degrees and in Screw fixation group was 100.1 degrees, which was statistically insignificant (P value-0.2506). However there were 11 cases $(27.5 \%)$ that had significant reduction in the range of motion (arc less than 100 degrees); in both the groups. The mean range of pronation in the TBW group was found to be 79.5 degrees, (standard deviation 7.76) while the mean range of pronation in the Screw fixation group was found to be 78degrees, (standard deviation 6.37). The mean range of supination in the TBW group was found to be 73.5 degrees, (standard deviation 11.01) while the mean range of supination in the Screw fixation group was found to be 72 degrees, (standard deviation9.79). Both were found to be statistically insignificant.

The overall average MEPS for 40 transverse olecranon fractures receiving operative treatment by either method were82.25. For the tension band wiring group mean MEPS was 85.25with a range from 65 to 100 (standard deviation: 09.662)and that for Screw fixation group was 79.25 with a range from 50 to 100 (standard deviation: 15.413). On statistical analysis p value was found out to be 0.150 , which is statistically insignificant. 


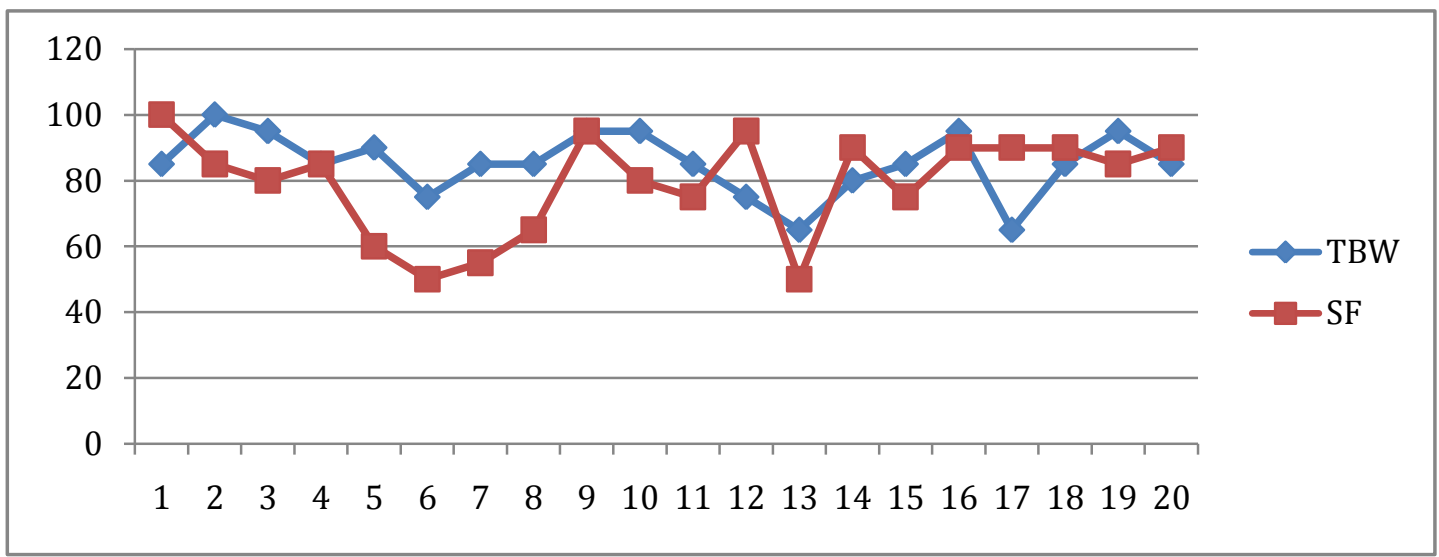

In our study overall satisfactory result (excellent+ good) was obtained in $82.5 \%$ cases. In the TBW group satisfactory result were obtained in $90 \%$ cases whereas in Screw fixation group it was obtained in $75 \%$ cases. The difference in outcome was found to be statistically insignificant (pvalue-0.6827)

The average time taken for union in tension band wiring group was 10.15 weeks and that for screw fixation was 10.6 weeks. On statistical analysis $p$ value was found out to be 0.4783 depicting no significant difference between the two techniques.

On follow up we found 3 cases with superficial infection till this period. Of them 2 were in TBW and 1 was in screw fixation. There were no cases with deep infection.Hardware prominences were observed in 3 cases of Tension band wiring. However, such complications didn't affect the union of the olecranon in either of the subjects.Non-union was seen in one particular case of tension band wiring treatment undergone.

\section{Discussion}

Olecranon fractures are relatively common upper limb injuries. In this study, we documented the longterm results of olecranon fracture for 40 patients. There have been a lot of controversies concerning the most appropriate management of fixation of transverse olecranon fractures. In this present study conducted at Gauhati Medical College and Hospital, we tried to correlate our clinical, functional and radiological results of both the procedures with other standard previous publications.

The criteria of Anderson et al (1975) were taken into account to assess the union of the fracture. Accordingly, all of the fractures in both the study groups united without the need for a second procedure, before 8 months with an exception in TBW, where non-union occurred. Thus the union rate was $97.5 \%$ in both the groups in the study.

The time taken for union in the TBW group ranged from 6 to 13 weeks with a mean of 10.15 weeks. The time taken in the Screw fixation group for radiological union ranged from 8 to 14 weeks, with a mean of 10.6weeks. The two groups were tested using the unpaired t test to bring about the presence of a significant difference, if any between the means of time for union in the two groups. The p value was 0.4873 which is considered not significant and shows that time for union does not depend on the type of implant used. Thus our study is comparable to most of the studies.

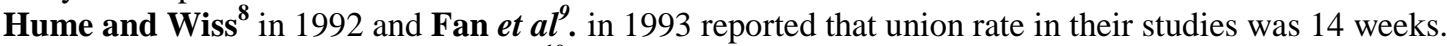

According to Wolfgang G et al. $1987^{\mathbf{1 0}}$, tension-band wiring usually provides stable fixation with a high union rate for simple non-comminuted transverse olecranon fractures.

Papagelopoulosn and Morrey, $\mathbf{1 9 9 4}^{\mathbf{1 1}}$ reported nonunion of olecranon fractures up to $1 \%$ of patients, with typical symptoms of pain, instability, or loss of motion.

The functional results were evaluated according to Mayo Elbow Performance scoring system (MEPS). With a maximum possible score of 100, the results are graded as excellent for scores $>90$; good for scores 75-89; fair for 60-74 and poor for scores less than 60 .

Although different authors employed different modes of internal fixation, the functional evaluation criteria were common in most of them.In this study we obtained excellent results $(37.5 \%)$ similar by previous authors Ahmed et al (40\%).However Satisfactory results were obtained in $82.5 \%$ of cases in our study which is almost similar to that obtained by Ahmed et al $^{12}$. Chalidis et al. ${ }^{13}$

In our study we achieved similar results with respect to mean flexion, mean arc of motion and mean supination pronation arc compared to earlier studies. Our findings are consistent with those stated by Chalidiset $a{ }^{13}$ and Lindenhoviuset al. ${ }^{14}$ and somewhat superior to that of Van der Linden et al. ${ }^{15}$ in terms of range of motion. Moreover flexion contracture or extension loss was found to somewhat similar as compared to most of the other studies. 


\section{Conclusion}

From this study conducted on 40 patients of transverse olecranon fractures, we came to the conclusion that operative treatment is the treatment for all kinds of olecranon fractures. Tension band wiring and parallel screw fixation are equally effective method of fixation in terms of union and elbow movements. If done perfectly, patient's postoperative satisfaction was found to be more in the screw fixation group. Minor complications can occur in both the groups equally.

\section{References}

[1]. Rommens PM, Schneider RU, Reuter M. Functional results after operative treatment of olecranon fractures. ActaChir Belg. 2004; 104 (2):191-7.

[2]. Court-Brown CM, Koval KJ. The Epidemiology of Fractures. In: BucholzRW, Heckman JD, Court- Brown C, editors. Rockwood and Green's Fractures in Adults. 6th ed. Lippincott, Williams \& Wilkins; 2005. p. 95-144.

[3]. Villanueva P, Osorio F, Commessatti M, Sanchez-Sotelo J. Tension-band wiring for olecranon fractures: analysis of risk factors for failure. Journal of shoulder and elbow surgery/ American Shoulder and Elbow Surgeons. 2006; 15:351-356.

[4]. Morrey BF. Current concepts in the treatment of fractures of the radial head, the olecranon and the coronoid. Journal of Bone and Joint Surgery. 1995; 77A:316-327.

[5]. Pun WK, Chow SP, So YC, Luk KD, Ip FK, Chan KC, Ngai WK, Crosby C, Ng C. A prospective study on 284 digital fractures of the hand. J Hand Surg [Am]1989;14:474-481.

[6]. Black D, Mann RJ, Constine R, Daniels AU. Comparison of internal fixation techniques in metacarpal fractures. J Hand Surg [Am] 1985; 10: 466-472.

[7]. Tyler B. Jones, BS, Andrew R. Karenz, MD, Paul S. Weinhold, PhD, and Laurence E. Dahners, MD; Transcortical Screw Fixation of the Olecranon Shows Equivalent Strength and Improved Stability Compared With Tension Band Fixation; J Orthop Trauma Volume 28, Number 3, March 2014.

[8]. Hume MC, Wisse DA. Olecranon fractures - a clinical and radiographic comparison of tension band wiring and plate fixation. ClinOrthopRelat Res 1991;229-35.

[9]. Fan G, Wu CC, Shin CH. Olecranon fractures with tension band wiring techniques - comparison among three different configurations. Chang Gung Medical Journal. 1993;16:231- 238.

[10]. Wolfgang G, Burke F, Bush D et al (1987) Surgical treatment of displaced olecranon fractures by tension band wiring technique.Clin Orthop 224:192-204

[11]. Papagelopoulos PJ, Morrey BF. Treatment of nonunion of olecranon fractures. J Bone Joint Surg 1994; 76B:627-635.

[12]. Ahmed AR, Sweed T, Wanas A. The role of cancellous screw with tension band fixation in the treatment of displaced Olecranon fractures, a comparative study. European Journal of Orthopaedic Surgery \& Traumatology. 2008;18:571-576.

[13]. Chalidis BE, Sachinis NC, Samoladas EP, Dimitriou CG, Pournaras JD. Is tension band wiring technique the "gold standard" for the treatment of olecranon fractures? A long term functional outcome study. Journal of orthopaedic surgery andresearch. 2008; 3:9.

[14]. Lindenhovius ALC, Brouwer KM, Doornberg JN et al. Long-term outcome of operatively treated fracture-dislocations of the olecranon. J Orthop Trauma 2008;22:325-31.

[15]. Van der Linden SC, van Kampen A, Jaarsma RL. K-wire position in tension-band wiring technique affects stability of wires and long-term outcome in surgical treatment of olecranon fractures. Journal of shoulder and elbow surgery/American Shoulder and Elbow Surgeons.2012;21:405-411. 